\title{
Early Dynamic Orchestration of Immunologic Mediators Identifies Multiply Injured Patients who are Tolerant or Sensitive to Hemorrhage
}

\author{
Todd O. McKinley, MD ${ }^{1}$ \\ tmckinley@iuhealth.org \\ Greg E. Gaski, MD ${ }^{2}$ \\ greg.gaski@inova.org \\ Ruben Zamora, $\mathrm{PhD}^{3}$ \\ zamorar@pitt.edu \\ Li Shen, $\mathrm{PhD}^{4}$ \\ li.shen@pennmedicine.upenn.edu \\ Qing Sun, $\mathrm{PhD}^{5}$ \\ qing.sun@usc.edu \\ Rami A. Namas MD ${ }^{3}$ \\ namasra2@upmc.edu \\ Timothy R. Billiar, MD ${ }^{3}$ \\ billiartr@upmc.edu \\ Yoram Vodovotz, $\mathrm{PhD}^{3}$ \\ vodovotzy@upmc.edu \\ ${ }^{1}$ Indiana University School of Medicine, Indianapolis, IN \\ ${ }^{2}$ INOVA Health System, Fairfax VA \\ ${ }^{3}$ University of Pittsburgh School of Medicine, Pittsburgh, PA \\ ${ }^{4}$ University of Pennsylvania School of Medicine, Philadelphia, PA \\ ${ }^{5}$ University of Southern California School of Medicine
}

This is the author's manuscript of the article published in final edited form as:

McKinley, T. O., Gaski, G. E., Zamora, R., Shen, L., Sun, Q., Namas, R. A., Billiar, T. R., \& Vodovotz, Y. (2021). Early Dynamic Orchestration of Immunologic Mediators Identifies Multiply Injured Patients who are Tolerant or Sensitive to Hemorrhage. Journal of Trauma and Acute Care Surgery, Publish Ahead of Print. https://doi.org/10.1097/TA.0000000000002998 
Corresponding Author

Todd O. McKinley

IU Health Orthopaedic Trauma Division

Methodist Hospital

1801 North Senate Boulevard Suite 535

Indianapolis, IN 46202

317 -963-1966

Fax 317-963-1955

tmckinley@iuhealth.org

Conflict of Interest: None of the authors have any conflicts of interest with the content of this manuscript.

Conference Presentations

$135^{\text {th }}$ Annual Meeting of the Orthopaedic Trauma Association. September $25^{\text {th }}-$ $28^{\text {th }}$ in Denver, $\mathrm{CO}$.

$217^{\text {th }}$ International Conference on Complexity of Acute Illness. September $26^{\text {th }}-$ 27th in Denver, CO. 
Background: Multiply injured patients are at risk of complications including infections, acute and prolonged organ dysfunction. The immunologic response to injury has been shown to affect outcomes. Recent advances in computational capabilities have shown that early dynamic coordination of the immunologic response is associated with improved outcomes after trauma. We hypothesized that patients who were sensitive or tolerant of hemorrhage would demonstrate differences in dynamic immunologic orchestration within hours of injury.

Methods: We identified two groups of multiply injured patients that demonstrated distinct clinical tolerance to hemorrhage $(n=10)$ or distinct clinical sensitivity to hemorrhage $(n=9)$ from a consecutive cohort of 100 multiply injured patients. Hemorrhage was quantified by integrating elevated shock index values for 24 hours after injury (Shock Volume). Clinical outcomes were quantified by average Marshall Organ Dysfunction Scores (MODS) from days two to five (aMODS $\left.\mathrm{D2}_{\mathrm{D}-\mathrm{D} 5}\right)$ after injury. Shock Sensitive (SS) patients had high cumulative organ dysfunction after lower magnitude hemorrhage. Shock Tolerant (ST) patients had low cumulative organ dysfunction after higher magnitude hemorrhage. Computational methods were used to analyze a panel of twenty immunologic mediators collected serially over the initial 72 hours after injury.

Results: Dynamic Network Analysis demonstrated the ST patients had increased orchestration of cytokines that are reparative and protective including interleukins 9, 17E/25, 21, 22, 23 and 33 during the initial $0-8 \mathrm{hr}$ and $8-24 \mathrm{hr}$ intervals after injury. SS patients had delayed immunologic orchestration of a network of largely pro- and anti-inflammatory mediators. Elastic Net Linear Regression demonstrated that a group of five mediators could discriminate between SS and ST 
patients.

Conclusions: Preliminary evidence from this study suggest that early immunologic orchestration discriminates between patients who are notably tolerant or sensitive to hemorrhage. Early orchestration of a group of reparative/protective mediators was amplified in Shock Tolerant patients.

Study Type: Original Article Prospective Clinical Outcomes Study

\section{Level of Evidence: III}

Keywords: Hemorrhagic shock; Immunologic response; Dynamic network analysis; Trauma tolerance; Trauma sensitivity 


\section{Introduction}

Multiply injured patients (MIPs) are at risk of developing complications. Typically, complications are related to the magnitude of injury and hemorrhage. For example, in recent work the magnitude of cumulative hypoperfusion corresponded to organ dysfunction, duration of mechanical ventilation and nosocomial infections. [1] However, there are anecdotal examples of patients that recover uneventfully after major injury or conversely, are plagued with complications after less severe injury.

Outcomes after trauma and hemorrhagic shock (HS) are clearly affected by the immunologic response to injury. [2-6] The immunologic response is complex, with multidimensional temporal and spatial relationships among immune cells and the biochemical communication orchestrated by the cells primarily through various inflammatory mediators. The complexities of the response are highlighted by uniform failure in the clinical trauma arena to improve outcomes by mitigating individual immunologic mediators expressed after injury. [7] Recently, multiple studies have yielded novel insights into the trauma immunologic response by employing computational methods that can account for temporal and spatial networks of mediators. [2-6, 810] Rather than focusing on isolated mediators -- typically cytokines and chemokines -- several studies have shown that immunologic orchestration among mediators better corresponds to favorable vs. unfavorable outcomes. [8, 11, 12] Patients who demonstrate early immunologic coordination followed by subsequent dissipation of dynamic networks of mediators have more favorable outcomes. In contrast, patients with poor early network orchestration followed by networks that grow in complexity have poor outcomes and higher mortality. [2, 10] Delayed 
immunologic network formation is affected by the magnitude of injury severity $[4,5,8]$, but it is distinctly possible that tolerance to trauma is affected by individual capability to orchestrate a favorable coordinated immune response at the time of injury.

We hypothesized that differences in the composite immunologic response would stratify tolerance to trauma and hemorrhagic shock (HS). We explored this concept in a prospective cohort of MIPs. Specifically, we identified two demographically similar groups of patients from a prospective cohort of 100 MIPs, that exhibited significant clinical tolerance or sensitivity to hemorrhage. Shock Tolerant (ST) patients had uncomplicated outcomes despite having increased cumulative hypoperfusion in the first $24 \mathrm{hr}$ after injury. In contrast, Shock Sensitive (SS) patients had poor outcomes despite having significantly less cumulative hypoperfusion during the same period. We hypothesized that there would be differences in individual circulating concentrations of immunologic mediators as well as distinct feature differences in dynamic networks of mediators between ST and SS cohorts. Our results identified two consistent clusters of immunologic mediators occurred in trauma patients. Furthermore, our results showed that SS patients had delayed overall mediator orchestration, delayed formation of a distinct mediator cluster associated with tissue repair and protection, and time-dependent increases in mediator network connectivity in a second cluster of inflammatory mediators. In contrast, ST patients had robust early mediator orchestration, specific orchestration primarily within the tissue protective/reparative cluster, and dissolution of mediator connectivity over time.

\section{Methods}

Patient Population: This study was approved by our Institutional Review Board. We 
prospectively enrolled 100 blunt trauma MIPs (Figure 1). Patients were 18 to 55 years old and met the following criteria: 1) presented as a full trauma activation defined by the General Surgical trauma team with the attending surgeon present at the initial resuscitation; 2) were admitted to surgical ICU or proceeded directly to surgery and were then admitted to surgical ICU. We excluded patients with non-survivable or severe traumatic brain injuries (TBI; Glasgow Coma Score of $\leq 7$ at presentation with no improvement after 48 hours after injury).

Fifty-one of the original 100 patients had TBI of which 15 were excluded when their GCS remained $\leq 7$ after $48 \mathrm{hr}$. Three patients with spinal cord injuries were also excluded. One additional patient sustained an iatrogenic air embolus with cardiac arrest and was excluded. This yielded the final study cohort of 81 patients (Figure 1).

Shock Tolerance and Shock Sensitivity. The purpose of this study was to compare immunologic profiles in patients that demonstrated wide discrepancy in tolerating hemorrhage. We compared the magnitude of hemorrhage to the magnitude of organ dysfunction to stratify individual tolerance/sensitivity to hemorrhage. Cumulative hypoperfusion during the first $24 \mathrm{hr}$ after injury was used to define the magnitude of hemorrhage. [1, 13] Previously, we demonstrated that cumulative hypoperfusion, measured by temporal integration of abnormally elevated shock index values over the first $24 \mathrm{hr}$ after injury corresponded closely with transfusion requirements and organ dysfunction (Figure 2). [1, 13] The cumulative hypoperfusion index, 24hr Shock Volume (24hr SHVL), demonstrated significantly greater correspondence with outcomes including organ dysfunction compared to Injury Severity Score (ISS) and Base Deficit (BD). Notably, organ dysfunction, nosocomial infections (NI) and transfusions increased abruptly in patients with $24 \mathrm{hr}$ 
SHVL $\geq$ to a threshold of 100 units. The magnitude of organ dysfunction was calculated by using serial Marshall Organ Dysfunction Scores (MODS). Previously we demonstrated that MODS averaged from days two to five after injury, aMODS ${ }_{\mathrm{D} 2-\mathrm{D} 5}$, identified patients at risk for prolonged ICU length of stay (ICU Days), prolonged mechanical ventilation (MV Days) and NI. [1, $11,12]$ In this work, there was a stark threshold of $\mathrm{aMODS}_{\mathrm{D2}-\mathrm{D} 5}>4$ that predicted poor outcomes.

From the cohort of 81 patients, we defined ST patients as those with aMODS ${ }_{\mathrm{D} 2-\mathrm{D} 5} \leq 4$ and $24 \mathrm{hr}$ SHVL $\geq 100$ (Figure 1, $\mathrm{n}=10$; Figure 2 red dashed box). In contrast, SS patients were defined by an $\operatorname{aMODS}_{\mathrm{D2}-\mathrm{D} 5}>4$ and 24hr SHVL $<100$ (Figure 1, n = 9; Figure 2 black dashed box). Initially, injury severity and demographics of the ST and SS groups were determined including ISS, age, gender, and Glasgow Coma Score (GCS). Subsequently, both groups were closely studied to identify more granular discrepancies in injury characteristics that may have accounted for differences in outcomes. Specifically, medical records were scrutinized to evaluate all preexisting comorbidities, trauma related diagnoses, the magnitude, type and resolution of TBI, the magnitude of initial hemorrhage, metabolic response to hemorrhage, transfusions, surgical interventions and mechanism of injury.

Cytokine and Chemokine Measurements. Serial panels of 20 cytokines, chemokines and high mobility group box one (HMGB1) were measured using a multiplex platform (Luminex ${ }^{\mathrm{TM}}$; Luminex Corporation, Austin, TX). The following inflammatory mediators were measured: Interleukin (IL)-1 $\beta$, IL-1 receptor antagonist (IL-1RA), soluble IL-2 receptor- $\alpha$ (sIL-2R $\alpha$ ), IL-4, IL-5, IL-6, IL-7, IL-8, IL-9, IL-10, IL-17A, IL-17E/IL-25, IL-21, IL-22, IL-23, IL-33, interferon 
gamma-induced protein 10 (IP-10), monokine induced by interferon gamma (MIG), monocyte chemoattractant protein-1 (MCP-1), and HMGB1. Mean values of individual mediators were calculated. Plasma was collected at the time of admission ( $0 \mathrm{hr})$, and subsequently at $8,24,48$ and $72 \mathrm{hr}$ after admission. Blood samples were all processed within two hours of collection. Samples were centrifuged at room temperature at $1500 \mathrm{rpm}$ for 10 minutes. $1.0 \mathrm{ml}$ of plasma was aliquoted into separate cryovial tubes and immediately frozen at -80 degrees Celsius.

Computational Modeling and Analyses: Two distinct analyses were conducted to evaluate immunologic orchestration and patterns of mediators. Time-dependent changes in mediator connectivity were measured using Dynamic Network Analysis (DyNA). [4, 14]. Discriminant Analysis [15-18] paired with Elastic Net Linear Regression (ENLR) [19] was used to identify cytokine networks that discriminated the ST and SS groups and the time windows in which the distinguishing networks were most evident.

Dynamic Network Analysis: These analyses explored temporal changes in network connectivity and complexity of the post-traumatic inflammatory response between the ST and SS groups. We have used DyNA in multiple studies which specifically detail the methods. [2, 9, 14] Inflammatory mediator networks were created in the sampling intervals $(0-8 \mathrm{hr} ; 8-24 \mathrm{hr} ; 24-48$ hr and 48-72 hr) using MATLAB ${ }^{\circledR}$ (The MathWorks, Inc., Natick, MA) as we have done previously $[2,9,14,20]$. Connections in the network were created if the correlation coefficient between two nodes (inflammatory mediators) was greater or equal to a threshold of 0.85 . For the network density calculation, in order to account for network sizes (number of significantly altered nodes) in the adjacent time periods detailed above, we utilized the following formula: 
Total number of edges $*$ Number of total nodes

Maximum possible edges among total nodes

Discriminant Analysis with Elastic Net Linear Regression. We performed a set of discriminant analyses with several widely used statistical and machine learning methods to examine the predictive power of the biomarkers in differentiating the ST and SS groups. Initially, the biomarkers were analyzed based on 1) individual cytokines concentrations at each discreet time point $(0,8,24,48$ and $72 \mathrm{hr})$; and 2) differences in each cytokine between adjacent time points (0-8 hr; 8-24 hr; 24-48 hr and 48-72 hr). Multivariate discriminant analyses of the above biomarker measures were examined between the SS and ST cohorts. Pattern classification was performed with four widely used machine learning methods including 1) Support Vector Machine learning using either Linear (Linear SVM) or Radial Basis Function (RBF SVM) as its kernel [15, 16]; 2) Decision Tree analyses [17]; and 3) Random Forest Modeling [18]. Classification performance was evaluated by leave-one-out cross-validation accuracy. Briefly, one observation was omitted and the classifier was learned from the remaining n-1 observations. The classifier was then applied to the hold out observation and the accuracy was recorded. This was repeated for all observations, and the average of all the recorded accuracies was used to evaluate the classification performance. Based on these analyses, we determined that the $0-8 \mathrm{hr}$ difference values between cytokines yielded the best cross-validation accuracy. Accordingly, we applied ENLR analyses [19] to 0-8 hr cytokine difference values to identify networks of biomarkers that best discriminated between ST and SS cohorts. 
ENLR is a sparse learning model including both the least absolute shrinkage and selection operator (LASSO) and ridge regularizations. By adjusting a model parameter balancing the LASSO and ridge effects, we identified the 11 most predictive cytokines with varying sparsity levels ranging from one relevant cytokine through all eleven cytokines respectively. To evaluate the power of the selected cytokines, we applied the above four classification methods (i.e., Linear SVM, RBF SVM, Decision Tree, and Random Forest) using only the eleven identified cytokines as predictors and estimated the leave-one-out cross-validation accuracy. These focused analyses were conducted only on the eleven identified cytokines and we compared differences between SS vs ST patients.

All the above machine learning analyses were implemented using Python with its libraries including NumPy, Pandas and Scikit-learn, and were performed on a desktop running Ubuntu 18.04 with Python3.6 installed.

Statistical Analyses for Clinical Data. Continuous clinical and demographical data were compared with paired Student's t-tests or Analysis of Variance (ANOVA) when appropriate. Categorical data were compared by Chi-Squared analyses.

\section{Results}

Organ dysfunction correlated with cumulative hypoperfusion. ST and SS groups were identified as outliers from regression analyses. Significant correspondence $\left(\mathrm{R}^{2}=0.49\right)$ was demonstrated between $24 \mathrm{hr}$ SHVL and aMODS ${ }_{\mathrm{D} 2-\mathrm{D} 5}$ from the entire cohort of 81 patients (Figure 2). Two outlier groups were observed including a group of ten ST patients with $24 \mathrm{hr}$ SHVL $\geq 100$ and 
$\operatorname{aMODS}_{\mathrm{D} 2-\mathrm{D} 5} \leq 4$ (red dashed box, Figure 2), and nine SS patients with $24 \mathrm{hr}$ SHVL $<100$ and $\operatorname{aMODS}_{\mathrm{D} 2-\mathrm{D} 5}>4$ (black dashed box, Figure 2).

Demographics, Injury Severity, Injury Distribution and Surgical Interventions were homogenous between SS and ST patients. There were no differences in age or gender between SS patients and ST patients (Table 1). The mean ISS for SS and ST patients was 32.0 (range 9 to 50; s.d. = 13.2) and 29.6 (range 21 to 48 ; s.d. $=8.6 ; \mathrm{p}=0.65$ ) and the majority of patients in both groups were injured in motor vehicle collisions (Table 1). Demographics, injury severity and mechanism of injury from both SS and ST patients reflected the overall cohort of 81 patients.

Injury distribution and surgical interventions were similar between the two experimental groups (Table 1). SS patients had more spine injuries in contrast to ST patients who had more abdominal and extremity injuries, however none of the differences were significant. Surgical interventions were similar between groups and reflected differences in spine and abdominal injuries. Granular details demonstrated no substantial differences in the initial magnitude and resolution of TBI between ST and SS patients (Table 1).

Clinical Outcomes reflect higher organ dysfunction and resource utilization in SS patients compared to ST patients (Supplemental Table 1, http://links.lww.com/TA/B832). Compared to ST patients, SS patients had a 5-fold increase in the $\mathrm{MV}_{\text {Days }}(\mathrm{p}<0.01)$ and a 3-fold increase in $\operatorname{ICU}_{\text {Days }}(\mathrm{p}<0.001)$. Likewise, SS patients had a greater incidence of NI $(\mathrm{p}=0.011)$ and a 2.3 fold increase in $\mathrm{aMODS}_{\mathrm{D} 2-\mathrm{D} 5}(\mathrm{p}<0.001)$ compared to ST patients. Individual organ dysfunction trajectories confirmed that ST patients (red lines Figure 3) resolved organ dysfunction primarily 
between hospital days 2 and 3, compared to SS patients which had little resolution of organ dysfunction from the time of injury to day five (black lines Figure 3).

Hemorrhage based outcomes demonstrated trends toward more bleeding in ST patients but no differences in anaerobic metabolism (Supplemental Table 2, http://links.lww.com/TA/B833). Bleeding indices, serum measurements of anaerobic metabolism and transfusion requirements in the first $24 \mathrm{hr}$ demonstrated that ST patients had greater 24hr SHVL (by definition of the study design) which is reflected in significantly higher HR $(\mathrm{p}<0.01)$ and a trend toward greater Shock Index $(p=0.11)$ at the time of injury compared to SS patients. However, there were no differences in anaerobic indices (BD and $\mathrm{pH}$ ) during the initial 0-24 $\mathrm{hr}$ after injury between the groups. Initial Hgb was $0.8 \mathrm{~g} / \mathrm{dl}$ lower in ST patients compared to SS patients but the difference was not significant. Four of ten ST patients had a critical transfusion requirements [21] (three or more units of PRBCs transfused within a 60 minute period) compared to two of nine patients in the SS group. Likewise, three of ten of the ST patients had a massive transfusion (10 or more units of PRBCs in a $24 \mathrm{hr}$ period) compared to one of nine SS patients. With the small numbers of patients, differences in the incidence of Massive Transfusion and Critical Administration Transfusions were not significant. Likewise, there were no statistical differences in mean units of packed red blood cell (PRBCs), platelet, and plasma (FFP) transfusions between the groups. However, mean 0-24 hr transfusion values in SS patients were largely influenced by a single patient that received a massive transfusion. There were minimal transfusions in either group after the first $24 \mathrm{hr}$ (Supplemental Table 2, http://links.lww.com/TA/B833). In summary, ST patients had greater cumulative hypoperfusion and tachycardia, but no evidence of increased anaerobic metabolism compared to SS patients. 
There were minimal differences in individual mediator concentrations between the SS and ST patients at any time point. Differences in individual mediators between the groups were scattered (Supplemental Figure 1, http://links.lww.com/TA/B831) but the only individual mediator that was significantly different between the two groups was HMGB1 which was higher in SS patients. None of the cytokines or chemokines were different between the two groups.

Two distinct clusters of mediators were observed in both experimental groups. DyNA quantifies coordination between individual mediators within a time interval. Two clusters of mediators were consistently observed in DyNA in both groups (Figure 4; Supplemental Figure 2, http://links.lww.com/TA/B830). The first cluster included eleven cytokines, primarily associated with pro and anti-inflammatory functions [22, 23], including IL-1 $\beta$, IL-17A, IL-1RA, IL-4, IL-5, IL-6, IL-7, IL-8, IL-10, IP-10 and MCP-1 (Cluster One; delineated in the black dashed boxes in Figure 4). The second cluster had six cytokines including IL-9, IL-21, IL-22, IL-23, IL-33 and IL-17E/25 (Cluster Two; red dashed boxes Figures 4) that have been associated with reparative functions and boundary organ protection. [24-26]

SS patients had reduced overall early dynamic mediator orchestration and delayed coordination of Cluster Two compared to ST patients. In the first 0-8 $\mathrm{hr}$ interval, there were only five overall mediator connections in SS patients compared to eleven connections in ST patients (Figure 4). Overall DyNA connections increased to fourteen connections in the 8-24 hr interval (Figure 4) in SS patients which primarily reflected robust development of Cluster One (Figure 4 black dashed box). Conversely, in the $0-8 \mathrm{hr}$ and $8-24 \mathrm{hr}$ intervals, SS patients had only three DyNA 
connections between mediators in Cluster Two (Figure 4 red dashed boxes). In contrast, ST patients had early and robust dynamic orchestration of Cluster Two (Figure 4 red dashed boxes) forming seven connections in the $0-8 \mathrm{hr}$ interval which expanded to twelve connections in the 8$24 \mathrm{hr}$ interval. In the 8-24 hr interval, IL-21 and IL-9 were both connected to five other mediators and IL-17E/25 and IL-33 formed four connections. Interestingly, in the 48-72 $\mathrm{hr}$ interval, there was complete dissolution of all connectivity in ST patients.

SS patients had greater connectivity of Cluster One compared to ST patients. SS patients developed a highly orchestrated network involving Cluster One (Figure 4 black dashed box) in the 8-24 hr interval forming eleven overall connections. MCP-1 was connected to five other mediators. IL-1 $\beta$, IL-6 and IL-10 were connected to four other mediators. In contrast, ST patients formed only six connections in Cluster One in the 8-24 hr interval and MCP-1 formed no connections (Figure 4 black dashed box).

Discriminant Analyses and Elastic Net Linear Regression demonstrated that mediator differences between $0 \mathrm{hr}$ and $8 \mathrm{hr}$ were most discriminating between SS and ST patients. Discriminant analyses demonstrated that both Random Forest Modeling and Decision Tree Analysis were superior in identifying mediators that best distinguished ST from SS patients (Table 2). In addition, the highest discriminant values were consistently measured by modeling the $0-8 \mathrm{hr}$ mediator difference values.

ENLR (Table 2) identified a hierarchical order of mediators that best distinguished ST and SS patients. MIG was the single most distinguishing mediator between SS and ST patients. Immune 
response differences between SS and ST patients were statistically distinguished by including 0$8 \mathrm{hr}$ differences in five mediators (in descending order of influence) including MIG, sIL-2RA, IL-23, MCP-1 and IL-1RA $\left(\mathrm{R}^{2}=0.191 ; \mathrm{p}=0.021\right)$. However, this initial model accounted for only $19 \%$ of the variance between the two groups. Adding $0-8 \mathrm{hr}$ changes in IL-6, IP-10 and IL22 to the model nearly doubled the discriminating power between ST and SS patients with an $\mathrm{R}^{2}$ value of 0.368 . Finally, incremental increases in regression correspondence were quantified with the addition of IL-10 and IL-17E/IL-25.

\section{Discussion}

The data from this experiment demonstrated differences in the immunologic response in patients identified as ST compared to patients identified as SS. We used computational approaches to account for the complexity of the immunologic response after injury. The most distinguishing features of the immunologic response between the two groups demonstrated that SS patients had overall reduced dynamic immunologic orchestration in the initial 0-8 hr interval, ST patients had greater immunologic orchestration in the first $24 \mathrm{hr}$ after injury involving a distinct cluster of protective/reparative cytokines, SS patients had greater orchestration of a second distinct cluster of pro/anti-inflammatory cytokines, and in the latest 48-72hr interval ST patients had complete dissipation of all immunologic orchestration. Additionally, the single most distinguishing mediator between SS and ST groups identified from ENLR analyses, MIG, did not fit into either Cluster but formed a single connection with Cluster One in SS patients (Figure 4). All observations need to be appropriately tempered by the small number of patients and the pathomechanistic significance of any of the observations is unknown. 
Cluster One contains cytokines that have been associated with pro-inflammatory (IL-1 $\beta$, IL-5, IL-6, IL-8, IP-10 and MCP-1) and anti-inflammatory (IL-1RA, IL-4, IL-10) functions. [22, 23] Cluster Two is comprised of cytokines that have substantial tissue protective/reparative effects. [24-26] Cluster Two cytokines are particularly protective of barrier organs including the skin, lung and gut all of which have been shown to be significantly compromised by injury. [25, 26] Interestingly, concentrations of IL-17E/25, IL-21, IL-23 and IL-33 were higher in survivors of blunt trauma at the time of admission compared to non-survivors. Furthermore, prehospital administration of plasma led to early increases in IL-17E/25, IL-21, IL-23 and IL-33 and increased survival compared to untreated patients. [27] In another retrospective report, IL-33 was elevated in blunt trauma survivors compared to a propensity matched group of non-survivors. [28]

Researchers have established that the immunologic response to injury plays a major and potentially dominant role in acute outcomes. [2, 4-6, 9, 11, 22, 23] Likewise, it is increasingly recognized that immunologic dysfunction affects longer-term outcomes after injury. [29, 30] Numerous studies have quantified association between immunologic mediators and outcomes, [31-35] however causation models linking individual mediator changes with postinjury phenotypes are notably absent. Accordingly, researchers are increasingly leveraging computational methods to understand how injury incites and propagates the immunologic response and how the response affects outcomes. [2, 4, 9, 14, 36, 37] For example, Abboud and colleagues demonstrated distinct immunologic feature differences, using DyNA, in blunt trauma survivors and non-survivors in closely matched cohorts. Survivors had early orchestration of predominantly lymphoid-based cytokines. Non-survivors had greater innate immunity-based 
cytokine networks that were initially delayed and then expanded in complexity over a 72-hour time frame. [2] In another study, patients with poor outcomes after subarachnoid hemorrhage had reduced initial cytokine orchestration with delayed progressive orchestration of cytokine networks that included MCP-1, IL-6 and IL-1RA (Cluster One cytokines). Conversely, in patients with good outcomes, network orchestration was early and IL-9 (Cluster Two cytokine) played a central role in mediator networks in survivors. [36] In summary, computational capabilities in trauma-based immunologic studies have uncovered consistent themes that 1) increasing injury severity uncouples early immunologic coordination; 2) early immunologic orchestration is associated with improved outcomes; and 3) patients with reduced initial immunologic orchestration followed by delayed expansion of immunologic network connectivity are at risk for poor outcomes. Methodologic advancements will be necessary to develop immunologic assay platforms and computational methods that can quantify individual immunologic networks at the time of injury to inform clinical decisions and interventions.

Our data are preliminary, and the experimental groups are small which could clearly affect the results. Accordingly, clinical extrapolation of these results is not possible. For example, transfusions in a single patient in the SS group more than doubled the mean values of transfused units of PRBCs, platelets and FFP within the SS group. Likewise, while there were more spine injuries in SS patients and more abdominal injuries in ST patients (Table 1), with the small group numbers the differences were not significant. It is possible that these differences may have affected the results. However, three of the four SS patients who had spine surgery did so within 36 hours of injury and there were no additional transfusions associated with these three surgeries. Our findings will need to be validated in an expanded prospective trial. Our definition of ST and 
SS is admittedly arbitrary. However, in our foundational studies [1, 13] cumulative hypoperfusion was more accurate than ISS and BD in predicting outcomes. Other components specific to the injury and specific to the patient may better account for the clinical differences between the ST and SS cohorts. Transfusions were reported only for the initial $24 \mathrm{hr}$ in these groups. However, there were minimal transfusions in either group after the first $24 \mathrm{hr}$ (Supplemental Table 2, http://links.lww.com/TA/B833). We scrutinized injury-associated and demographic variables to identify alternative explanations for the clinical disparities between the two groups and found no meaningful differences (Table 1). Collectively, it is unlikely that clinical differences were attributable to demographics, injury magnitude and distribution, or interventions. Our analyses are singularly focused on the immunologic response to injury. We utilized two different computational methods to provide an in-depth exploration of the immunologic response at distinct cross-sections in the injury time frame and dynamically during progression of injury. However, it is possible that other global response mechanisms to injury such as metabolic response were more critical determinants of clinical outcomes.

In summary, from a larger cohort of MIPs, two selected sub-cohorts that had ST and SS clinical trajectories demonstrated fundamentally different computational immunologic responses. Increased early orchestration in cytokine networks corresponded to improved outcomes. In particular, early dynamic orchestration of a reparative/protective cluster of cytokines was increased in ST compared to SS patients. Larger populations of patients need to be interrogated to explore this model. 
Authorship: T.O.M., G.E.G., T.R.B. and Y.V. participated in study design, data collection and analysis, statistical analysis and manuscript preparation. R.Z., L.S., Q.S. and R.A.N participated in data collection and analysis, and manuscript preparation.

Conflict of Interest: None of the authors have any conflict of interest pertaining to this work.

Funding Sources. This work was supported by funding received from the Department of Defense Combat Casualty Care Research Program (Joint Program Committee-6) and the Major Extremity Trauma and Rehabilitation Consortium (METRC) funded through the Department of Defense Peer Reviewed Orthopaedic Research Program. 


\section{References}

1. McKinley TO, McCarroll T, Metzger C, Zarzaur BL, Savage SA, Bell TM, Gaski GE. Shock volume: Patient-specific cumulative hypoperfusion predicts organ dysfunction in a prospective cohort of multiply injured patients. J Trauma Acute Care Surg. 2018;85(1S Suppl 2):S84-S91.

2. Abboud A, Namas RA, Ramadan M, Mi Q, Almahmoud K, Abdul-Malak O, Azhar N, Zaaqoq A, Namas R, Barclay DA, et al. Computational Analysis Supports an Early, Type 17 Cell-Associated Divergence of Blunt Trauma Survival and Mortality. Crit Care Med. 2016;44(11):e1074-e81.

3. Abdul-Malak O, Vodovotz Y, Zaaqoq A, Guardado J, Almahmoud K, Yin J, Zuckerbraun B, Peitzman AB, Sperry J, Billiar TR, et al. Elevated Admission Base Deficit Is Associated with a Complex Dynamic Network of Systemic Inflammation Which Drives Clinical Trajectories in Blunt Trauma Patients. Mediators Inflamm. 2016;2016:7950374.

4. Almahmoud K, Namas RA, Abdul-Malak O, Zaaqoq AM, Zamora R, Zuckerbraun BS, Sperry J, Peitzman AB, Billiar TR, Vodovotz Y. Impact of Injury Severity on Dynamic Inflammation Networks Following Blunt Trauma. Shock. 2015;44(2):101-9.

5. Almahmoud K, Namas RA, Zaaqoq AM, Abdul-Malak O, Namas R, Zamora R, Sperry J, Billiar TR, Vodovotz Y. Prehospital Hypotension Is Associated With Altered Inflammation Dynamics and Worse Outcomes Following Blunt Trauma in Humans. Crit Care Med. 2015;43(7):1395-404.

6. Namas RA, Almahmoud K, Mi Q, Ghuma A, Namas R, Zaaqoq A, Zhu X, Abdul-Malak O, Sperry J, Zamora R, et al. Individual-specific principal component analysis of circulating inflammatory mediators predicts early organ dysfunction in trauma patients. J Crit Care. 
2016;36:146-53.

7. Spruijt NE, Visser T, Leenen LP. A systematic review of randomized controlled trials exploring the effect of immunomodulative interventions on infection, organ failure, and mortality in trauma patients. Crit Care. 2010;14(4):R150.

8. Almahmoud K, Abboud A, Namas RA, Zamora R, Sperry J, Peitzman AB, Truitt MS, Gaski GE, McKinley TO, Billiar TR, et al. Computational evidence for an early, amplified systemic inflammation program in polytrauma patients with severe extremity injuries. PloS one. 2019;14(6): $\mathrm{e} 0217577$.

9. Namas RA, Vodovotz Y, Almahmoud K, Abdul-Malak O, Zaaqoq A, Namas R, Mi Q, Barclay D, Zuckerbraun B, Peitzman AB, et al. Temporal Patterns of Circulating Inflammation Biomarker Networks Differentiate Susceptibility to Nosocomial Infection Following Blunt Trauma in Humans. Ann Surg. 2016;263(1):191-8.

10. Schimunek L, Namas RA, Yin J, Liu D, Barclay D, El-Dehaibi F, Abboud A, Lindberg H, Zamora R, Billiar TR, et al. An Enrichment Strategy Yields Seven Novel Single Nucleotide Polymorphisms Associated With Mortality and Altered Th17 Responses Following Blunt Trauma. Shock. 2018;49(3):259-68.

11. Gaski GE, Metzger C, McCarroll T, Wessel R, Adler J, Cutshall A, Brown K, Vodovotz Y, Billiar TR, McKinley TO. Early Immunologic Response in Multiply Injured Patients With Orthopaedic Injuries Is Associated With Organ Dysfunction. J Orthop Trauma. 2019;33(5):220-8.

12. Lamparello AJ, Namas RA, Constantine G, McKinley TO, Elster E, Vodovotz Y, Billiar TR. A conceptual time window-based model for the early stratification of trauma patients. $J$ Intern Med. 2019;286(1):2-15. 
13. McKinley TO, McCarroll T, Gaski GE, Frantz TL, Zarzaur BL, Terry C, Steenburg SD. Shock Volume: A Patient-Specific Index That Predicts Transfusion Requirements and Organ Dysfunction in Multiply Injured Patients. Shock. 2016;45(2):126-32.

14. Mi Q, Constantine G, Ziraldo C, Solovyev A, Torres A, Namas R, Bentley T, Billiar TR, Zamora R, Puyana JC, et al. A dynamic view of trauma/hemorrhage-induced inflammation in mice: principal drivers and networks. PloS one. 2011;6(5):e19424.

15. Cortes C, Vapnik V. Support Vector Networks. Machine Learning. 1995;20(3):273 - 97.

16. Hocking RR. A Biometrics Invited Paper. The Analysis and Selection of Variables in Linear Regression. Biometrics. 1976;32(1):1-49.

17. Quinlan JR. Induction of decision trees. Machine Learning. 1986;1(1):81-106.

18. Tin Kam H. The random subspace method for constructing decision forests. IEEE Trans Pattern Anal Mach Intell. 1998;20(8):832-44.

19. Zou H, Hastie T. Regularization and Variable Selection via the Elastic Net. J R Stat Soc Series B Stat Methodol. 2005;67(2):301-20.

20. Ziraldo C, Vodovotz Y, Namas RA, Almahmoud K, Tapias V, Mi Q, Barclay D, Jefferson BS, Chen G, Billiar TR, et al. Central role for MCP-1/CCL2 in injury-induced inflammation revealed by in vitro, in silico, and clinical studies. PLoS One. 2013;8(12):e79804.

21. Savage SA, Sumislawski JJ, Zarzaur BL, Dutton WP, Croce MA, Fabian TC. The new metric to define large-volume hemorrhage: results of a prospective study of the critical administration threshold. J Trauma Acute Care Surg. 2015;78(2):224-9; discussion 9-30.

22. Huber-Lang M, Lambris JD, Ward PA. Innate immune responses to trauma. Nat Immunol. 2018;19(4):327-41.

23. Lord JM, Midwinter MJ, Chen YF, Belli A, Brohi K, Kovacs EJ, Koenderman L, Kubes P, 
Lilford RJ. The systemic immune response to trauma: an overview of pathophysiology and treatment. Lancet. 2014;384(9952):1455-65.

24. Anthony RM, Rutitzky LI, Urban JF, Jr., Stadecker MJ, Gause WC. Protective immune mechanisms in helminth infection. Nat Rev Immunol. 2007;7(12):975-87.

25. Eyerich K, Dimartino V, Cavani A. IL-17 and IL-22 in immunity: Driving protection and pathology. Eur J Immunol. 2017;47(4):607-14.

26. Robinson KM, Ramanan K, Clay ME, McHugh KJ, Rich HE, Alcorn JF. Novel protective mechanism for interleukin-33 at the mucosal barrier during influenza-associated bacterial superinfection. Mucosal Immunol. 2018;11(1):199-208.

27. Gruen DS, Brown JB, Guyette FX, Vodovotz Y, Johansson PI, Stensballe J, Barclay DA, Yin J, Daley BJ, Miller RS, et al. Prehospital plasma is associated with distinct biomarker expression following injury. JCI Insight. 2020;5(8).

28. Billiar IM, Guardado J, Abdul-Malak O, Vodovotz Y, Billiar TR, Namas RA. Elevations in Circulating sST2 Levels Are Associated With In-Hospital Mortality and Adverse Clinical Outcomes After Blunt Trauma. J Surg Res. 2019;244:23-33.

29. Horiguchi H, Loftus TJ, Hawkins RB, Raymond SL, Stortz JA, Hollen MK, Weiss BP, Miller ES, Bihorac A, Larson SD, et al. Innate Immunity in the Persistent Inflammation, Immunosuppression, and Catabolism Syndrome and Its Implications for Therapy. Front Immunol. 2018;9:595.

30. Vanzant EL, Lopez CM, Ozrazgat-Baslanti T, Ungaro R, Davis R, Cuenca AG, Gentile LF, Nacionales DC, Cuenca AL, Bihorac A, et al. Persistent inflammation, immunosuppression, and catabolism syndrome after severe blunt trauma. J Trauma Acute Care Surg. 2014;76(1):21-9; discussion 9-30. 
31. Gebhard F, Pfetsch H, Steinbach G, Strecker W, Kinzl L, Bruckner UB. Is interleukin 6 an early marker of injury severity following major trauma in humans? Arch Surg. 2000;135(3):291-5.

32. Gouel-Cheron A, Allaouchiche B, Guignant C, Davin F, Floccard B, Monneret G, AzuRea G. Early interleukin-6 and slope of monocyte human leukocyte antigen-DR: a powerful association to predict the development of sepsis after major trauma. PloS one. 2012;7(3):e33095.

33. Sherry RM, Cue JI, Goddard JK, Parramore JB, DiPiro JT. Interleukin-10 is associated with the development of sepsis in trauma patients. J Trauma. 1996;40(4):613-6; discussion 6-7.

34. Svoboda P, Kantorova I, Ochmann J. Dynamics of interleukin 1, 2, and 6 and tumor necrosis factor alpha in multiple trauma patients. J Trauma. 1994;36(3):336-40.

35. Taniguchi M, Nakada TA, Shinozaki K, Mizushima Y, Matsuoka T. Association between increased blood interleukin-6 levels on emergency department arrival and prolonged length of intensive care unit stay for blunt trauma. World J Emerg Surg. 2016;11:6.

36. Ahn SH, Savarraj JPJ, Parsha K, Hergenroeder GW, Chang TR, Kim DH, Kitagawa RS, Blackburn SL, Choi HA. Inflammation in delayed ischemia and functional outcomes after subarachnoid hemorrhage. J Neuroinflammation. 2019;16(1):213.

37. Lamparello AJ, Namas RA, Abdul-Malak O, Vodovotz Y, Billiar TR. Young and Aged Blunt Trauma Patients Display Major Differences in Circulating Inflammatory Mediator Profiles after Severe Injury. J Am Coll Surg. 2019;228(2):148-60 e7. 


\section{Figure Legends}

Figure 1. Experimental Group mapping leading to the SS and ST groups. Nineteen patients were excluded from the original 100 enrollees. Note that 9/48 (18.8\%) of patients with $24 \mathrm{hr}$ SHVL < 100 were SS, and 10/33 (30.3\%) with $24 \mathrm{hr}$ SHVL $\geq 100$ were ST by the screening criteria.

Figure 2. Linear regression shows significant correspondence $\left(R^{2}=0.49\right)$ between organ dysfunction (aMODS $\mathrm{D}_{\mathrm{D} 2 \mathrm{D} 5}$ ) and cumulative hypoperfusion (24hr SHVL) in 81 multiply injured patients. Two outlier sub-cohorts were identified that had higher magnitude organ dysfunction with lower 24hr SHVL (Shock Sensitive (SS) black dashed box; $\left(\right.$ aMODS $_{\mathrm{D} 2-\mathrm{D} 5}>$ 4; $24 \mathrm{hr}$ SHVL $<100$ ) or lower magnitude organ dysfunction and higher 24hr SHVL (Shock Tolerant (ST) red dashed box; aMODS $\mathrm{D2- \textrm {D } 5} \leq 4 ; 24 \mathrm{hr}$ SHVL $\geq 100)$.

Figure 3. Individual daily MODS scores for SS (black lines) and ST (red lines) groups demonstrate divergence in organ dysfunction trajectories nearly uniformly occurred between days 2 and 3 after injury, with resolution of organ dysfunction in ST patients. (* denotes significant difference with $\mathrm{p} \leq 0.05$ between $\mathrm{SS}$ and $\mathrm{ST}$ groups)

Figure 4. DyNA plots at each time interval for SS and ST patients demonstrate distinguishing features in mediator orchestration and clustering. Individual cytokines are denoted by the red and yellow nodes on the periphery of each circle (an enlarged nodal map detailing each mediator is available in Supplemental Figure 2, http://links.lww.com/TA/B830). Two-way arrows between cytokine nodes denote they are connected within that time interval. Two clusters of coordinated 
mediators were consistently identified including Cluster One (black dashed boxes) of eleven cytokines including IL-10, IL-17A, IL-1RA, IL-4, IL-5, IL-6, IL-7, IL-8, IL-10, IP-10 and MCP1, and six cytokines in Cluster Two (red dashed boxes) that included IL-9, IL-21, IL-22, IL-23, IL-33 and IL-17E/25. The most notable features include early robust coordination of Cluster Two in ST patients in contrast to delayed coordination of Cluster Two in SS patients, highmagnitude orchestration in Cluster One in the 8-24 hr interval in SS patients, and complete dissolution of immunologic coordination in ST patients by the $48-72 \mathrm{hr}$ interval. ST patients maintained robust coordination of Cluster Two for the first $48 \mathrm{hr}$ after injury. Overall connectivity graphs demonstrate delayed connectivity in SS patients in the initial 0-8 hr interval compared to ST patients.

Supplemental Figure 1. Box and Whisker plots of all 20 mediators at all time points demonstrated no distinguishing features between ST and SS patients. IL-5 demonstrated the greatest magnitude difference between TS and TT patients but the differences were not significant. SS patients had significantly higher concentrations of HMGB1.

Supplemental Figure 2. Individual cytokines in the DyNA circles are detailed. 
Figure 1

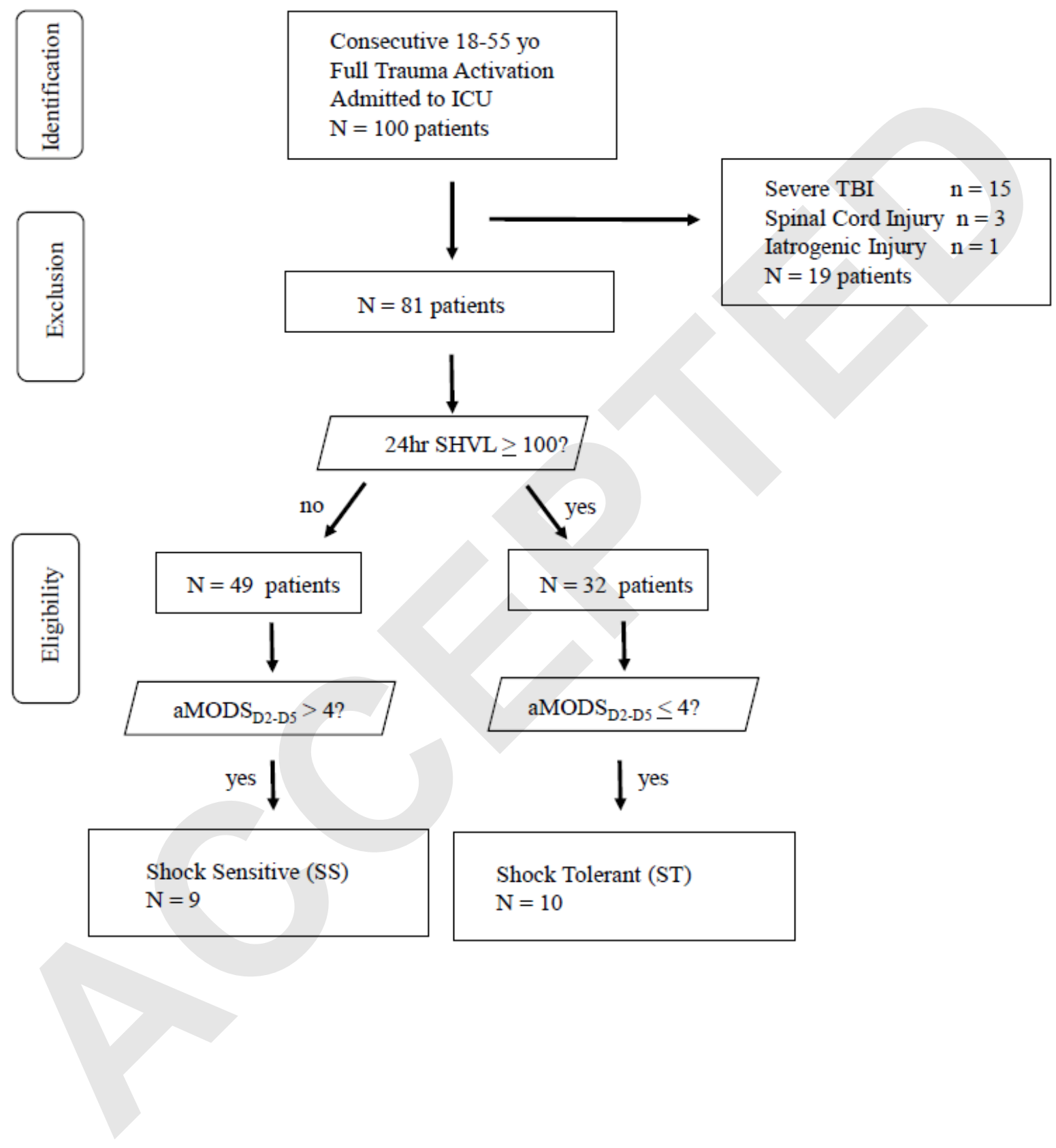


Figure 2

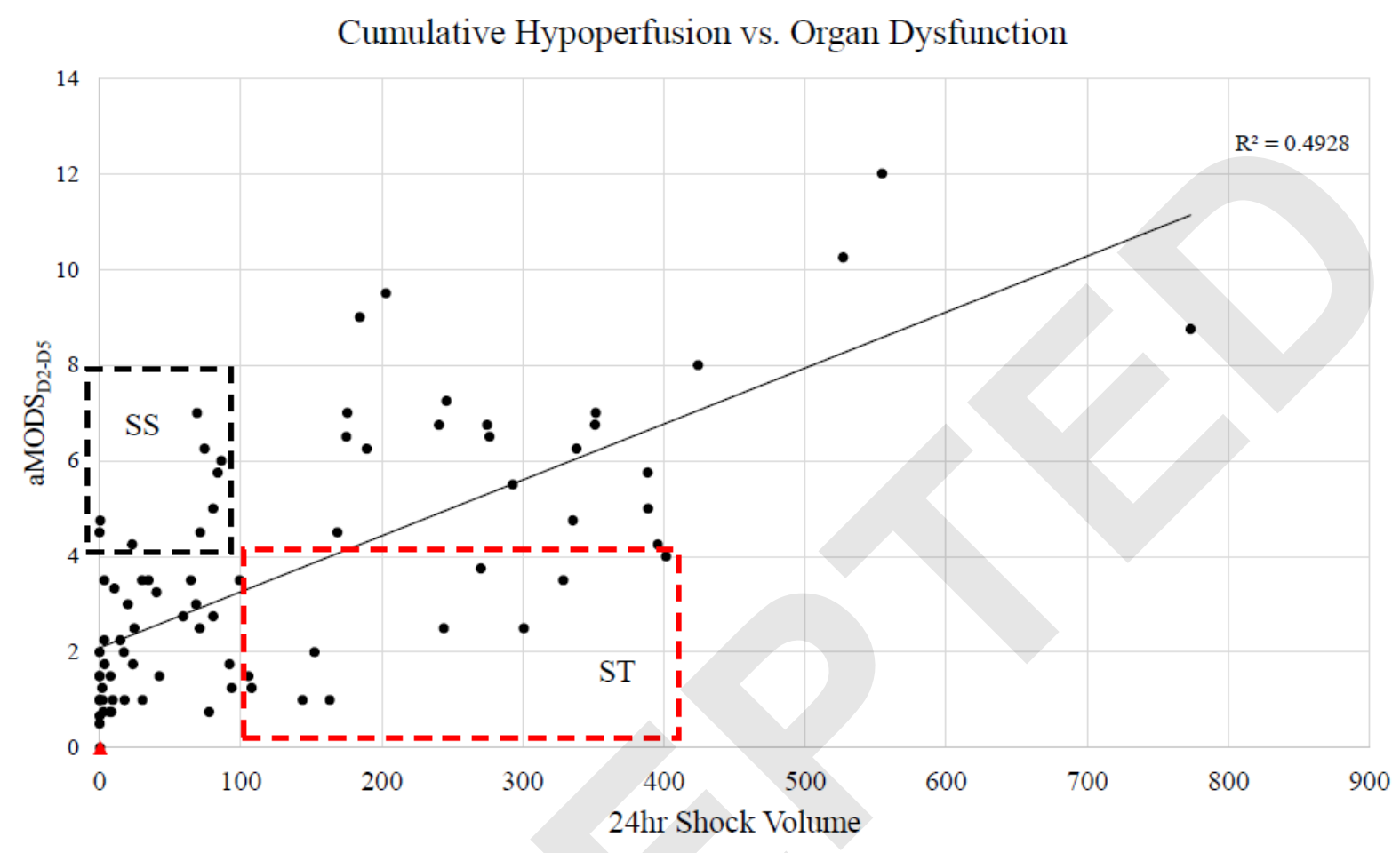


Figure 3

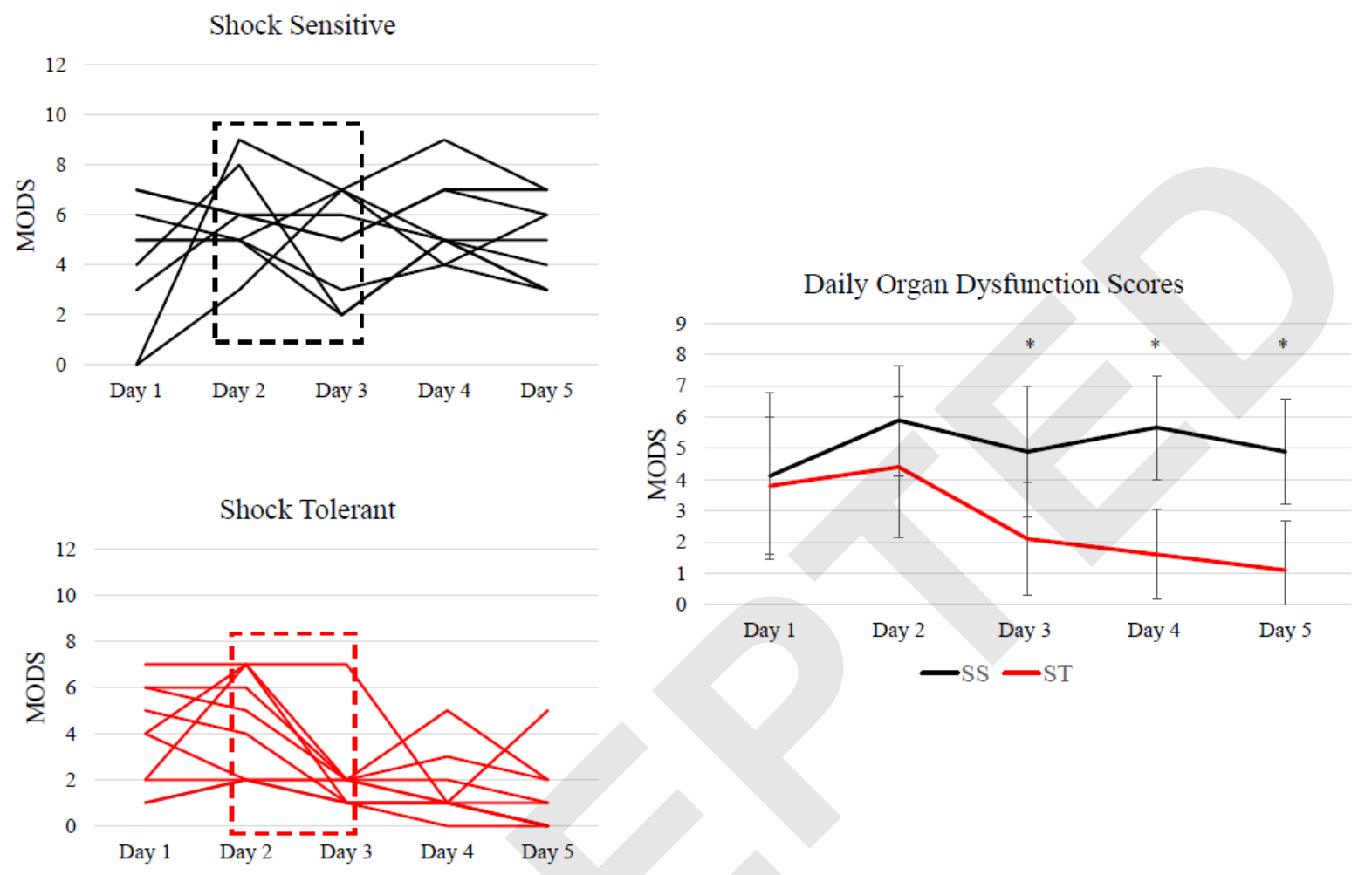


Figure 4

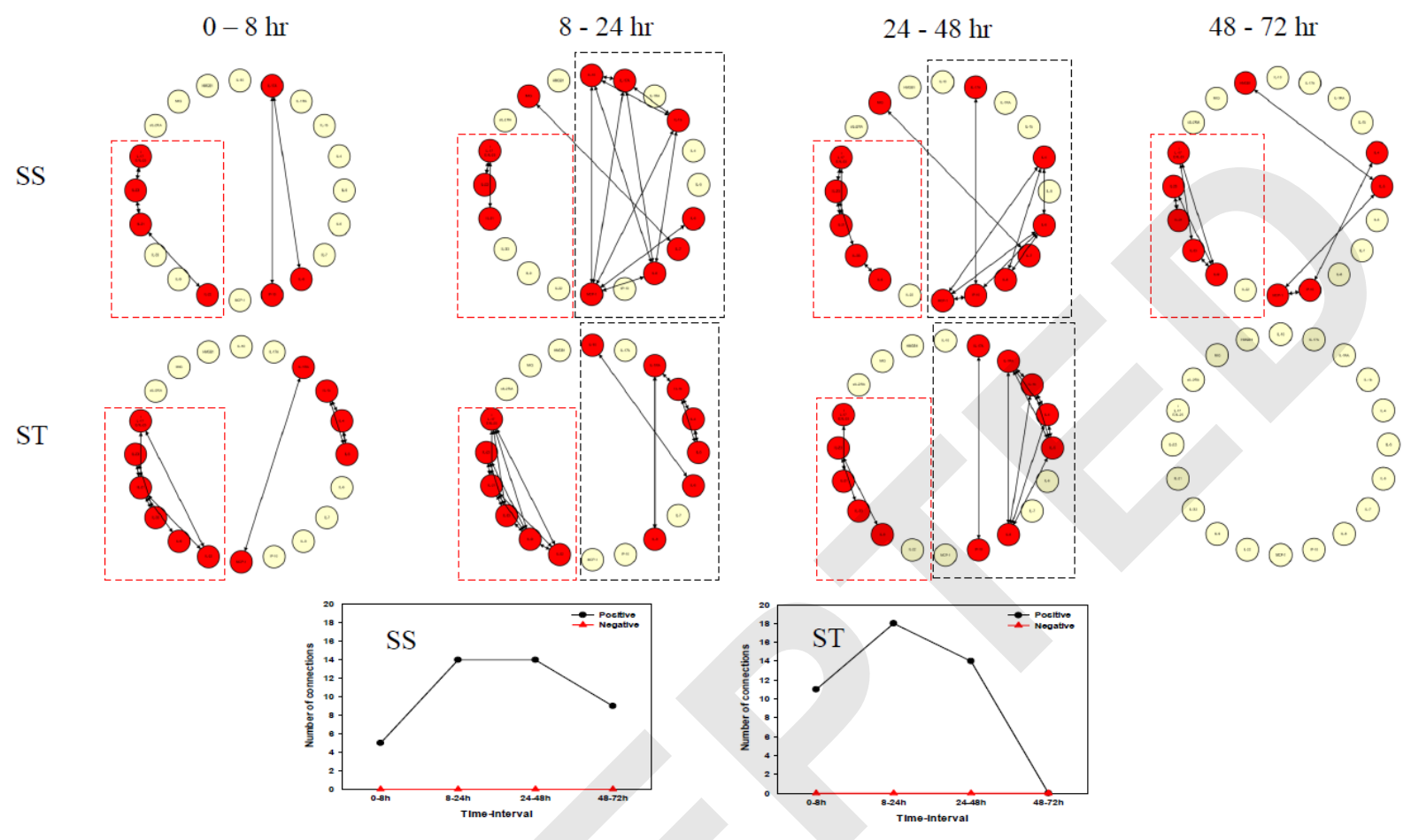


Table 1. Demographic variables, comorbidities, injury profiles, and surgical interventions.

\begin{tabular}{|c|c|c|c|c|}
\hline & $\begin{array}{l}\text { Entire } \\
\text { Cohort } \\
(n=81)\end{array}$ & $\begin{array}{c}\text { SS } \\
(\mathbf{n}=9)\end{array}$ & $\underset{(\mathbf{n}=10)}{\text { ST }}$ & $\begin{array}{c}p \text {-Value } \\
\text { (SS vs ST) }\end{array}$ \\
\hline \multicolumn{5}{|l|}{ Demographics, ISS, MVC } \\
\hline Age in years, Mean (SD) $\S$ & $36.6(11.4)$ & $33.7(12.5)$ & $36.6(11.8)$ & .61 \\
\hline Gender, Male/Female $\dagger$ & $60 / 21$ & $6 / 3$ & $7 / 3$ & .88 \\
\hline ISS, Mean (SD) $\S$ & $31.2(14.1)$ & $32.0(13.2)$ & $29.6(8.5)$ & .65 \\
\hline MVC, Yes/No ${ }^{\circ}$ & $49 / 32$ & $6 / 3$ & $7 / 3$ & .88 \\
\hline Comorbidities $\ddagger$ & & & & .17 \\
\hline Smoking & & 3 & 4 & \\
\hline Alcohol Abuse & & 1 & 1 & \\
\hline Diabetes & & 0 & 1 & \\
\hline COPD & & 1 & 1 & \\
\hline Cardiac Disease & & 0 & 0 & \\
\hline Liver Disease & & 0 & 0 & \\
\hline Kidney Disease & & 0 & 0 & \\
\hline Injury profilesł & & & & .92 \\
\hline $\mathrm{H} / \mathrm{N}$ & & 15 & 16 & \\
\hline Chest & & 33 & 20 & \\
\hline Abdomen & & 4 & 18 & \\
\hline Pelvis Retro & & 6 & 7 & \\
\hline Spine & & 14 & 2 & \\
\hline Extremity & & 10 & 22 & \\
\hline TBI: & & 4 & 4 & \\
\hline TBI Initial GCS, Mean (SD)§ & & $12.5(3.5)$ & $12.4(3.7)$ & .95 \\
\hline GCS 15, Yes/Not & & $5 / 4$ & $6 / 4$ & .84 \\
\hline$G C S \leq 8$, Yes/No $\dagger$ & & $2 / 7$ & $2 / 8$ & .91 \\
\hline TBI Final GCS, Mean $(S D) \S$ & & $15.0(0)$ & $15.0(0)$ & $>.99$ \\
\hline Surgical interventions & & & & .70 \\
\hline $\mathrm{H} / \mathrm{N}$ & & 1 & 0 & \\
\hline Chest & & 3 & 1 & \\
\hline Abdomen & & 0 & 3 & \\
\hline Pelvis/Retro & & 2 & 0 & \\
\hline Spine & & 4 & 0 & \\
\hline Lower extremity & & 5 & 10 & \\
\hline Upper extremity & & 2 & 7 & \\
\hline
\end{tabular}

SS, shock sensitive; ST, shock tolerant; $n$, number of patients; SD, standard deviation; ISS, injury severity score; MVC, motor vehicle crash; H/N, head and neck; TBI, traumatic brain injury; GCS, the Glasgow Coma Scale. $\S$ Student's t-test; $\uparrow$ Chi-square test; + ANOVA test. 
Table 2. Four methods intervals including Support Vector Machine (SVM) learning using Linear and Radial Based Functions, Random Forest Modeling and Decision Tree Analysis were used for discriminant analyses. Random Forest modeling and Decision Tree analyses using mediator difference values in the $0-8 \mathrm{hr}$ interval, depicted below (s.d. in parentheses) numerically provided the greatest distinguishing differences between SS and ST patients. Subsequently, Elastic Net Linear Regression modeling was used to sequentially build discriminating rosters of mediators. Once five cytokines including IL-1RA, MCP-1, IL-23, sIL-2RA, MIG were enrolled in the model, statistically significant discrimination was identified between SS and ST patients $(p=0.021)$ but predictive power was modest $\left(R^{2}=0.191\right)$. Addition of three additional mediators including IL-6, IL-22 and IP-10 nearly doubled correspondence to an $\mathrm{R}^{2}=0.368$.

\begin{tabular}{|c|c|c|c|c|c|c|}
\hline & $\begin{array}{c}\text { SVM } \\
\text { Linear } \\
\text { Mean } \\
(\mathrm{SD})\end{array}$ & $\begin{array}{c}\text { SVM } \\
\text { RBF } \\
\text { Mean } \\
\text { (SD) }\end{array}$ & $\begin{array}{c}\text { Random } \\
\text { Forest } \\
\text { Mean } \\
\text { (SD) }\end{array}$ & $\begin{array}{c}\text { Decision } \\
\text { Tree } \\
\text { Mean } \\
\text { (SD) }\end{array}$ & $\begin{array}{l}\text { Regression } \\
\mathrm{R}^{2}\end{array}$ & $\begin{array}{c}\text { T-test } \\
p \text {-Value }\end{array}$ \\
\hline $\begin{array}{l}\text { IL-10, IL-1RA, IL-6, IP-10, MCP-1, IL-22, IL- } \\
\text { 23, IL-17E/IL-25, sIL-2RA, MIG, HMGB1 }\end{array}$ & $\begin{array}{c}0.16 \\
(0.73)\end{array}$ & $\begin{array}{c}0.16 \\
(0.73)\end{array}$ & $\begin{array}{c}0.21 \\
(0.82)\end{array}$ & $\begin{array}{c}0.68 \\
(0.93)\end{array}$ & 0.487 & .001 \\
\hline $\begin{array}{l}\text { Il-10, IL-1RA, Il-6, IP-10, MCP-1, IL-22, IL-23, } \\
\text { IL-17E/IL-25, sIL-2RA, MIG }\end{array}$ & $\begin{array}{c}0.16 \\
(0.73)\end{array}$ & $\begin{array}{c}0.16 \\
(0.73)\end{array}$ & $\begin{array}{l}0.37 \\
(0.96)\end{array}$ & $\begin{array}{c}0.74 \\
(0.88)\end{array}$ & 0.458 & .001 \\
\hline $\begin{array}{l}\text { Il-10, IL-1RA, Il-6, IP-10, MCP-1, IL-22, IL-23, } \\
\text { sIL-2RA, MIG }\end{array}$ & $\begin{array}{c}0.16 \\
(0.73)\end{array}$ & $\begin{array}{c}0.16 \\
(0.73)\end{array}$ & $\begin{array}{c}0.21 \\
(0.82)\end{array}$ & $\begin{array}{c}0.74 \\
(0.88)\end{array}$ & 0.402 & .003 \\
\hline $\begin{array}{c}\text { IL-1RA, Il-6, IP-10, MCP-1, IL-22, IL-23, sIL- } \\
\text { 2RA, MIG }\end{array}$ & $\begin{array}{c}0.26 \\
(0.88)\end{array}$ & $\begin{array}{c}0.21 \\
(0.82)\end{array}$ & $\begin{array}{c}0.47 \\
(1.00)\end{array}$ & $\begin{array}{c}0.47 \\
(1.00)\end{array}$ & 0.368 & .006 \\
\hline $\begin{array}{c}\text { IL-1RA, IP-10, MCP-1, IL-22, IL-23, sIL-2RA, } \\
\text { MIG }\end{array}$ & $\begin{array}{l}0.21 \\
(0.82)\end{array}$ & $\begin{array}{c}0.21 \\
(0.82)\end{array}$ & $\begin{array}{c}0.47 \\
(1.00)\end{array}$ & $\begin{array}{c}0.53 \\
(1.00)\end{array}$ & 0.301 & .009 \\
\hline IL-1RA, IP-10, MCP-1, IL-23, sIL-2RA, MIG & $\begin{array}{c}0.26 \\
(0.88)\end{array}$ & $\begin{array}{c}0.16 \\
(0.73)\end{array}$ & $\begin{array}{c}0.53 \\
(1.00)\end{array}$ & $\begin{array}{c}0.74 \\
(0.88)\end{array}$ & 0.294 & .008 \\
\hline IL-1RA, MCP-1, IL-23, sIL-2RA, MIG & $\begin{array}{c}0.21 \\
(0.82)\end{array}$ & $\begin{array}{c}0.21 \\
(0.82)\end{array}$ & $\begin{array}{c}0.63 \\
(0.96)\end{array}$ & $\begin{array}{c}0.74 \\
(0.88)\end{array}$ & 0.191 & .021 \\
\hline MCP-1, IL-23, sIL-2RA, MIG & $\begin{array}{c}0.21 \\
(0.82)\end{array}$ & $\begin{array}{c}0.11 \\
(0.61)\end{array}$ & $\begin{array}{c}0.21 \\
(0.82)\end{array}$ & $\begin{array}{c}0.21 \\
(0.82)\end{array}$ & 0.046 & .372 \\
\hline MCP-1, IL-23, MIG & $\begin{array}{c}0.42 \\
(0.99)\end{array}$ & $\begin{array}{c}0.47 \\
(1.00)\end{array}$ & $\begin{array}{c}0.21 \\
(0.82)\end{array}$ & $\begin{array}{c}0.21 \\
(0.82)\end{array}$ & 0.045 & .377 \\
\hline IL-23, MIG & $\begin{array}{c}0.53 \\
(1.00)\end{array}$ & $\begin{array}{c}0.53 \\
(1.00)\end{array}$ & $\begin{array}{c}0.21 \\
(0.82)\end{array}$ & $\begin{array}{c}0.21 \\
(0.82)\end{array}$ & 0.035 & .436 \\
\hline MIG & $\begin{array}{c}0.53 \\
(1.00)\end{array}$ & $\begin{array}{c}0.53 \\
(1.00)\end{array}$ & $\begin{array}{c}0.11 \\
(0.61)\end{array}$ & $\begin{array}{c}0.11 \\
(0.61)\end{array}$ & 0.029 & .458 \\
\hline
\end{tabular}

SS, shock sensitive; ST, shock tolerant; SVM, support vector machine; RBF, radial basis function; SD, standard deviation; IL, interleukin; IL-1RA, the interleukin 1 receptor antagonist; IP-10, interferon gamma-induced protein 10; MCP-1, monocyte chemoattractant protein-1; sIL-2RA, soluble interleukin-2 receptor alpha; MIG, the monokine induced by interferon-gamma; HMGB1, high mobility group box 1 . 